\title{
Las estratégias de cluster en América Latina frente a los desafíos del cambio estrutural: experiencias recientes y contribuciones para una nueva agenda.
}

\author{
Néstor Bercovich, Prof. Depto. de Economia e Relações Internacionais, UFSC. \\ Silvio Cário, Prof. Depto. de Economia e Relações Internacionais, UFSC.
}

\section{Resumen}

Para retomar y hacer sustentables los procesos de crecimiento con inclusión social que varios países de América Latina protagonizaron en años recientes, será preciso que la región impulse profundos procesos de transformación productiva para ir superando su actual especialización basada en ventajas estáticas (recursos naturales) y para alcanzar niveles superiores de productividad. Las políticas industriales y tecnológicas son fundamentales para viabilizar ese proceso, y entre ellas se destacan las políticas de clústers. La experiencia reciente de la región pone en evidencia que estas últimas pueden hacer un aporte importante al proceso de cambio estructural, facilitando el desarrollo de capacidades competitivas y de innovación y viabilizando la superación de las brechas de productividad que caracterizan la estructura productiva latinoamericana. Una nueva y necesaria agenda de políticas clúster en la región debería sin embargo extraer algunas lecciones claves de aquella experiencia, en particular la necesidad de una fuerte articulación con las políticas nacionales y regionales que afectan la competitividad de los clústers. Las políticas clúster deben formar parte activa de estrategias más amplias de innovación regional.

Palabras claves: Cambio estructural, Políticas de clústers, Especialización inteligente.

\section{$\underline{\text { Resumo }}$}

Para retomar e tornar sustentáveis os processos de crescimento com inclusão social que vários países da América Latina experimentaram nos últimos anos, será necessário que a região promova significativos processos de transformação produtiva para superar sua atual especialização baseada em vantagens estáticas (recursos naturais) e para alcançar níveis mais altos de produtividade. Políticas industriais e tecnológicas são essenciais para viabilizar esse processo e, entre elas, as políticas de cluster. A experiência recente da região mostra que estas últimas podem contribuir de maneira importante para o processo de mudança estrutural, facilitando o desenvolvimento de capacidades competitivas e inovadoras e viabilizando a superação progressiva das brechas de produtividade que caracterizam a estrutura produtiva latino-americana. Uma nova e necessária agenda de políticas de clusters na região deve, no entanto, extrair algumas lições-chave dessa experiência, em particular a necessidade de uma forte articulação com políticas nacionais e regionais que afetam a competitividade dos clusters. As políticas de clusters devem fazer parte ativa de estratégias mais amplas de inovação regional.

Palavras-chave: Mudança estrutural, Políticas de Clústers, Especialização Inteligente. 


\begin{abstract}
To resume and make sustainable the processes of growth with social inclusion that several countries of Latin America played in recent years, it will be necessary for the region to promote deep processes of productive transformation to overcome its current specialization based on static advantages (natural resources) and to achieve higher levels of productivity. Industrial and technological policies are essential to make this process viable, and among these are cluster policies. The recent experience of the region shows that the latter can make an important contribution to the process of structural change, facilitating the development of competitive and innovative capacities and making it possible to overcome the productivity gaps that characterize the Latin American productive structure. A new and necessary cluster policy agenda in the region should nevertheless draw some key lessons from that experience, in particular the need for a strong articulation with national and regional policies that affect the competitiveness of clusters. Cluster policies need to be an active part of broader regional innovation strategies.

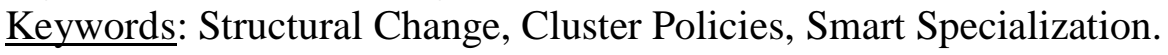

Área Abein:

Área 5.7 Inovação, competências e competitividade

Classificação JEL: O25.

\title{
1. Introducción
}

Brasil y la mayor parte de los países de América Latina atravesaron un período de crecimiento económico sostenido con creciente inclusión social entre principios de los años 2000 y principios de la presente década, en un contexto de términos de intercambio muy favorables que terminaron profundizando la especialización en recursos naturales de las economías de la región. El contexto económico y político cambió radicalmente a partir de 2013, incluyendo una progresiva reversión de los avances en igualdad del período previo. Retomar el camino del crecimiento con inclusión social y hacerlo en forma sustentable requerirá un cambio en el estilo de desarrollo y, en particular, avanzar hacia un cambio estructural que permita mejorar el patrón de especialización de nuestras economías y el despliegue de actividades productivas más intensivas en conocimiento que generen más empleo y de mejor calidad (CEPAL, 2016).

En ese sentido parece pertinente revisar la experiencia reciente de la región latinoamericana con políticas e instrumentos de desarrollo productivo e innovación, para reflexionar sobre su vigencia y posibles reformulaciones y así actualizar el menú de instrumentos más aptos que la región tiene a disposición para encarar su proceso de cambio estructural.

Un componente importante de las políticas de desarrollo productivo y regional de los primeros años 2000, en algunos países de la región y también a nivel internacional, han sido las políticas de clústers, que son el foco de análisis del presente artículo. 
En este artículo se hace una revisión de algunos balances disponibles de las políticas clúster implementadas en Brasil y la región latinoamericana y se propone una sistematización de dichas experiencias, orientada principalmente a reflexionar sobre su posible contribución al proceso de cambio estructural. También se presentan los marcos conceptuales e instrumentos desplegados por las iniciativas más recientes, tanto en América Latina como en Europa. Finalmente, sobre la base de la experiencia pasada, se discuten cuáles podrían ser los principales elementos que debería contemplar la nueva generación de estrategias clúster en el contexto del proceso de cambio estructural que debe encarar la región.

\section{Desafío de cambio estructural y estrategias clúster en América Latina}

Desde principios de los años 2000 y hasta 2012-13, Brasil y buena parte de los países latinoamericanos experimentaron un crecimiento económico sostenido y disminución de las desigualdades, gracias a la adopción de políticas centradas en la expansión de los mercados internos y la redistribución de ingresos, en un contexto de términos de intercambio favorables para la región. La región logró una importante reducción de la pobreza, del desempleo y de la desigualdad de ingresos, mejoras en la cobertura educativa y de protección social, avances en derechos y consolidación de la estabilidad económica. La CEPAL estimó que en 2013 la tasa de pobreza en América Latina fue de $27,9 \%$ de la población, frente al 43,9 \% registrado en 2002. La tasa de desempleo, en tanto, fue de 6,4\% en 2012, mientras que en 2002 alcanzaba al 11,2\% (CEPAL, 2016).

Sin embargo, esos progresos se estancaron y tienden a revertirse presentemente. A partir de 2013 la región experimentó una fuerte desaceleración económica, con epicentro en América del Sur. La presente coyuntura de bajo crecimiento se acompaña además de una reversión de los avances sociales: los objetivos de igualdad e inclusión social fueron perdiendo jerarquía en las agendas de políticas públicas de muchos países.

Siempre de acuerdo con la CEPAL, el porcentaje de hogares latinoamericanos con niveles mínimos de doble inclusión social y laboral, que había aumentado del 20,4\% en 2002 al 29,2\% en 2014, bajó al 28,6\% en 2015; en tanto que el porcentaje de hogares en doble exclusión, que había bajado del 44,1\% al 33,0\% entre 2002 y 2014, se elevó al 33,3\% en 2015 (CEPAL, 2017).

En buena medida, ese retroceso está relacionado con las restricciones externas generadas por la pérdida de dinamismo del comercio internacional tras la crisis de 20082009 y, en particular, el fin del boom de los precios de las materias primas a partir de 2014, que impactó directamente en el nivel de actividad, sobre todo en los países de América del Sur. Tampoco se puede desdeñar el impacto que tuvieron sobre ese retroceso los recientes y radicales cambios de orientación política en varios gobiernos latinoamericanos, que iniciaron reformas pro mercado.

Pero es evidente que la región enfrenta también limitaciones endógenas y estructurales para poder sostener los avances en materia económica y social logrados en la primera década del siglo. Aquí sobresalen los persistentes bajos niveles de productividad y de inversión, la limitada incorporación de progreso técnico, una estructura productiva excesivamente centrada en recursos naturales y bienes de escaso valor agregado y el déficit en la prestación de servicios públicos. A ello están asociados, entre otros 
problemas, las altas tasas de informalidad laboral que se observan en la región, las grandes desigualdades regionales y en la distribución de ingresos (CEPAL, 2018).

El punto es que sin un cambio radical en el actual patrón de producción y especialización internacional, intensivo en materias primas, que permita avanzar hacia una mayor diversificación productiva e incorporación de conocimiento y valor agregado, será muy difícil viabilizar y hacer sostenibles las agendas pro igualdad. Porque solamente ese cambio estructural es el que puede permitir un crecimiento sostenido, cerrar las brechas de productividad y generar más y mejor empleo, con mayores niveles de formalidad, calificación y remuneración.

Esta cuestión se torna aún más urgente debido a la revolución tecnológica e industrial en curso, que cambia dramáticamente las condiciones productivas y de competencia a escala internacional, donde al tiempo que emergen nuevos sectores y mercados se destruyen actividades y empleos que ya no responden al nuevo paradigma. La irrupción de la manufactura 4.0 en los países más industrializados, la difusión masiva de la automatización y la inteligencia artificial, entre otras tecnologías de creciente difusión, plantea un nuevo desafío a la región latinoamericana: el riesgo es un corrimiento dramático de la frontera tecnológica internacional, la pérdida de competitividad de industrias tradicionales y la destrucción masiva de empleos.

Las políticas industriales y tecnológicas serán claves para avanzar en dirección al cambio estructural. Porque los incentivos de mercado resultan a todas luces insuficientes para inducir el tránsito desde economías con clara especialización en actividades con ventajas comparativas estáticas (principalmente abundancia de recursos naturales) hacia otras que incorporen crecientemente actividades intensivas en conocimiento y con ventajas dinámicas.

En realidad, Brasil y varios países de la región implementaron políticas industriales y tecnológicas muy pro-activas en la primera década del 2000, en línea con las preocupaciones antes mencionadas. Sin embargo, en un contexto de términos de intercambio excepcionalmente favorables y una macroeconomía que no siempre favoreció procesos de transformación productiva, las inversiones en el período se concentraron mayormente en el área de recursos naturales y nuestros países tendieron a profundizar su especialización comercial tradicional. Hay que considerar, además, que los procesos de catch up y transformación productiva requieren largos períodos de maduración y por ello es crucial que las políticas e instituciones que respaldan ese proceso consigan amplios consensos y sean sustentables.

Para retomar el sendero de una estrategia de desarrollo socialmente inclusivo, habrá entonces que poner en el centro de la agenda pública el objetivo de la transformación productiva, con políticas industriales y tecnológicas acordes y políticas macro y comerciales consistentes con ese objetivo.

Un componente importante de las políticas de desarrollo productivo y regional de las últimas dos décadas, en la región y también a nivel internacional, han sido las políticas de clústers. Si bien el surgimiento de estos sistemas productivos especializados y localizados no es un fenómeno reciente, las estrategias para promover y potenciar a los clústers se difundieron y tomaron ímpetu importante en las últimas dos décadas en los países industrializados, y algo más recientemente en los menos avanzados. 
La contribución positiva de los clústers a la innovación, competitividad e internacionalización de las empresas allí localizadas, y en particular de las Pymes, ha sido ampliamente documentada en la literatura económica (Becattini, 2002; Porter, 1998; Schmitz y Nadvi, 1999; Pietrobelli y Rabellotti, 2007; Boscherini y Poma, 2000; Mattos et al, 2017). Fenómenos como las externalidades "Marshallianas" generadas en las aglomeraciones industriales, así como la "eficiencia colectiva" descripta por Schmitz (1997) como resultado de la cooperación y de las acciones colectivas deliberadas tanto privadas como públicas, son mecanismos que favorecen los aprendizajes e interacciones entre las firmas localizadas en ambientes favorables, y en general el acceso a bienes "externos" a las firmas pero "internos" al clúster. Esto facilita la superación de las restricciones que enfrentan típicamente las Pymes para su desarrollo: limitado acceso a insumos y servicios de capacitación, información, tecnología, financiamiento y mercados externos.

Por lo tanto, como instrumentos probadamente aptos para impulsar la cooperación, la innovación y la competitividad empresarial - particularmente en Pymes-, las políticas de clústers pueden hacer una contribución importante al necesario proceso de cambio estructural que tiene ante sí ALC. También, en la medida que aquellas permitan el desarrollo de clústers innovadores y competitivos, podrán favorecer una mayor y mejor inserción de estos sistemas productivos especializados en el mercado internacional y en cadenas globales de valor.

Más allá de esa argumentación conceptual, es evidente también el impacto positivo que han tenido las recientes políticas de clústers en la región. Por un lado, su contribución al desarrollo de capacidades productivas y empleo en polos productivos de escaso dinamismo previo. Por otro, la relevancia adquirida por clústers en sectores intensivos en conocimiento, como por ej. los de Guadalajara (TIC) y Querétaro (aviación) en México, o también Recife - Porto Digital (TIC), Florianópolis (TIC) y Sao José dos Campos - Embraer (aviación) en Brasil, son todos elementos que permiten pensar que las estrategias clúster deberán ser un componente importante de las políticas industriales tendientes a la transformación productiva de nuestras economías.

De hecho varios países latinoamericanos -y Brasil en particular-, han desarrollado en años recientes importantes políticas de apoyo a clústers. A continuación se hace una reseña de los principales avances logrados y de las limitaciones encontradas, con énfasis en los temas más vinculados con las políticas de cambio estructural.

\section{Estrategias clúster en AL: avances y limitaciones}

Algunos países latinoamericanos implementaron, con particular énfasis a partir de inicios de los años 2000, políticas nacionales de apoyo a clústers. Entre las iniciativas más ambiciosas y estructuradas sobresalen las de Brasil (Política Nacional de Arranjos Produtivos Locais -APLs), Colombia (Rutas Competitivas, Red Clúster Colombia), Argentina (Programa de Aglomerados Productivos), Chile (Programa Nacional de Clústers y, recientemete, los Programas estratégicos de especialización inteligente), Uruguay (PACPYMES) y México (Agendas estatales y regionales de innovación). 
Nos proponemos presentar a continuación una sistematización de los diversos balances de la implementación de las estrategias clúster en AL, sus impactos, limitaciones y principales lecciones. Consideraremos para ello tres grandes fuentes de información:

a) Algunos de los principales estudios y evaluaciones realizadas en años recientes sobre la experiencia de Brasil, que es probablemente la política nacional de clúster más integral y sostenida aplicada en la región. Aquí se consultaron principalmente estudios realizados por un grupo de académicos vinculados a la "Rede de Pesquisa em Arranjos e Sistemas Produtivos e Inovativos Locais", RedeSist, que estuvieron muy involucrados en el diseño e implementación de esas políticas.

b) Las evaluaciones y análisis realizados por el Banco Interamericano de Desarrollo (BID), institución que brindó apoyo a programas clúster en varios países latinoamericanos durante los últimos años, y

c) La información disponible sobre políticas y clústers latinoamericanos resultante del proyecto "Polos de Competitividad", de la EU-LAC Foundation, en el cual participan principalmente Instituciones implementadoras de políticas clúster de diversos países de América Latina (https://eulacfoundation.org/es).

Como puede verse, las fuentes de información utilizadas provienen de Instituciones diversas y muy vinculadas al diseño e implementación de políticas clúster en la región latinoamericana.

\section{a. La experiencia de Brasil}

La experiencia de Brasil merece ser presentada aquí "in extenso" por haberse implementado a lo largo de una década como política nacional claramente jerarquizada dentro de la política nacional de desarrollo, por haber impactado varios centenares de aglomeraciones productivas, por haber involucrado un Grupo de Trabajo interministerial, Gobiernos regionales, Cámaras empresarias y Universidades, y por haber suscitado una enorme cantidad de estudios académicos.

\section{$\underline{\text { Impactos }}$}

- Un balance sobre las políticas para clústers (Arranjos Produtivos Locais -APLs-) en Brasil, realizado por un grupo de economistas de la UFRJ que estuvieron muy involucrados en el diseño e implementación de estas políticas, al abordar los impactos de las mismas concluye que la convergencia de las diferentes iniciativas y programas de apoyo a APLs desde el inicio de la década de 2000 han sido de gran importancia para los mismos, contribuyendo al desarrollo de capacidades productivas e innovadoras y al diseño de estrategias competitivas. Se reconoce que, en general, las políticas han alcanzado un éxito considerable en lo que se refiere a los avances en las técnicas de producción y de organización de las relaciones socioproductivas, además de contribuir a la expansión del empleo y de la generación de ingresos en muchas localidades (Matos et al, 2016). Esta correlación positiva entre desarrollo de clústers y empleo en Brasil fue además confirmada por estudios del BID (Cravo, Lodato y Piza, 2013).

- En este último sentido, otros estudios enfatizan también la gran cobertura territorial que tuvieron estas iniciativas y la capacidad aportada por las políticas de APLs de 
actuar sobre espacios normalmente marginados de las grandes políticas industriales. Asismismo, se destaca el avance en la coordinación entre los ámbitos nacional y estadual, lo que llevó a fortalecer los niveles locales de políticas y la construcción participativa y cooperativa de las iniciativas, permitiendo así tomar en cuenta las especificidades locales (Matos, Borin y Cassiolato, 2015; Matos, Cassiolato, Lastres, Lemos y Szapiro (2017).

- En términos de contribución al cambio estructural, se destaca el aporte de estas políticas especialmente para al desarrollo y modernización de polos productivos en actividades tradicionales y en regiones poco atendidas por las grandes políticas industriales. Esto fue consistente con el objetivo de disminución de desigualdades sociales y regionales de las políticas nacionales de desarrollo implementadas en el período, y marcó un avance en el camino de superación de la heterogeneidad estructural y de las brechas de productividad. Al mismo tiempo, varios APLs más consolidados en sectores priorizados por la política industrial también pudieron beneficiarse de los programas de apoyo generales contemplados en la política nacional APL (Cassiolato y Matos, 2012).

- Aunque en las distintas evaluaciones no surgen evidencias claras que indiquen un desempeño innovador diferencial de las empresas localizadas en APLs en comparación con el resto del universo empresarial, en general en todas las evaluaciones se reconoce la intensificación de las actividades colectivas de capacitación y de vinculación con instituciones de conocimiento.

\section{Limitaciones}

- Los estudios examinados para el caso de Brasil enfatizan que el impacto de las políticas de clústers no pueden disociarse del contexto de políticas y del sistema económico y competitivo más general. Se reconoce en ese sentido que muchos APLs tuvieron sus desempeños condicionados no solamente por sus dinámicas internas sino también por determinantes exógenos (déficit de infraestructura, tipo de cambio, presión importadora, etc).

- Vinculado con lo anterior, se reconoce ampliamente un importante grado de desconexión de las políticas nacionales de APLs en relación con las políticas de desarrollo regional, por un lado, pero también respecto a otras políticas nacionales relevantes como las industriales, C\&T y de infraestructura (Lastres, Pietrobelli, Caporali, Soares, Matos, 2012).

- Los estudios antes mencionados sugieren que la rápida diseminación de la política nacional de APLs como resultado de los incentivos ofrecidos, llevaron a una asimilación bajo el concepto de "APL" de diversas iniciativas de apoyo prevalentes, de corte general, que en realidad no seguían formatos innovadores ni pertinentes para el desarrollo de clústers, es decir, no incorporaban una lógica territorial, colaborativa y sistémica.

- También surge de dichos estudios que los criterios de selección de los APLs a ser apoyados variaron de acuerdo con los diversos criterios empleados en las instancias nacional/estadual/local. Pero en general la política nacional parece haber priorizado APLs con actividades tradicionales intensivas en mano de obra, con fuerte presencia de micro y pequeñas empresas y en áreas relativamente periféricas. En ese sentido los estudios aquí considerados concluyen que muchas de las iniciativas asumieron un rol compensatorio, de inclusión social y generación de empleo, llevando apoyos a territorios/actividades que no se beneficiaban de las grandes políticas industriales sectoriales. 
- Los APLs parecen no haber conseguido superar la típica restricción que enfrentan en general las Pymes para el acceso al crédito, más allá del avance general de la oferta de crédito para Pymes durante el período considerado.

- Muchas iniciativas se concentraron exclusivamente en la organización/consolidación de una gobernanza para los APLs, y no pudieron avanzar en la implementación de actividades más generales de apoyo al desarrollo productivo de los APLs.

- Aunque el cuadro general que presentan los balances evidencian una intensificación de la articulación y coordinación entre actores dentro de los APLs, paradójicamente las prácticas estrictas de cooperación entre empresas no parecen haberse expandido significativamente dentro de los aglomerados.

- Finalmente, muchos APLs no consiguieron mantener su dinámica una vez interrumpidos o restringidos los mecanismos de apoyo contemplados por las políticas.

$\underline{\text { Aspectos claves de la experiencia con políticas APLs en Brasil }}$

\begin{tabular}{|c|c|c|}
\hline Impactos & Limitaciones & Lecciones \\
\hline $\begin{array}{l}\text { Aumento de capacidades } \\
\text { productivas e innovadoras }\end{array}$ & $\begin{array}{l}\text { Desconexión con políticas de } \\
\text { desarrollo regional y políticas } \\
\text { nacionales }\end{array}$ & $\begin{array}{l}\text { No pertinencia de modelos } \\
\text { estándar de políticas debido a } \\
\text { diferencias por sector/región, etc }\end{array}$ \\
\hline Mejora en empleo e ingresos & $\begin{array}{l}\text { Sobreposición/confusión con } \\
\text { iniciativas previas sin eje } \\
\text { territorial/colaborativo/sistémico }\end{array}$ & $\begin{array}{l}\text { Superar fragmentación y } \\
\text { descoordinación con políticas } \\
\text { más generales de desarrollo }\end{array}$ \\
\hline $\begin{array}{l}\text { Mayor coordinación ámbitos } \\
\text { nacional-estaduales }\end{array}$ & $\begin{array}{l}\text { Heterogeneidad del sentido de } \\
\text { las intervenciones entre los } \\
\text { niveles nacional/estadual/local, } \\
\text { actuando muchas veces con una } \\
\text { función compensatoria de } \\
\text { inclusión social }\end{array}$ & $\begin{array}{l}\text { El impulso de proyectos que } \\
\text { beneficien al APL no debe } \\
\text { subordinarse al objetivo de } \\
\text { consolidación de la gobernanza }\end{array}$ \\
\hline $\begin{array}{l}\text { Intensificación relaciones entre } \\
\text { empresas e instituciones de } \\
\text { conocimiento }\end{array}$ & $\begin{array}{l}\text { Los APLs no permitieron } \\
\text { superar las dificultades de } \\
\text { acceso al crédito enfrentadas por } \\
\text { las Pymes, y no consiguieron } \\
\text { grandes avances en cooperación } \\
\text { empresarial }\end{array}$ & $\begin{array}{l}\text { La movilización y capacitación } \\
\text { de una masa crítica de actores } \\
\text { del APL es clave para garantizar } \\
\text { su autonomía y la } \\
\text { sustentabilidad en el tiempo de } \\
\text { las iniciativas. }\end{array}$ \\
\hline Gran cobertura territorial & $\begin{array}{l}\text { Muchas iniciativas se } \\
\text { concentraron en afianzar el } \\
\text { núcleo de gobernanza sin llegar } \\
\text { a generar proyectos para el APL }\end{array}$ & 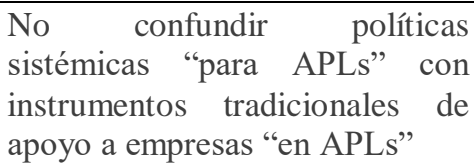 \\
\hline $\begin{array}{l}\text { Actuación sobre espacios } \\
\text { tradicionalmente marginados de } \\
\text { políticas industriales }\end{array}$ & $\begin{array}{l}\text { Muchos APLs no consiguieron } \\
\text { mantener su dinámica una vez } \\
\text { desactivadas las políticas de } \\
\text { apoyo }\end{array}$ & \\
\hline
\end{tabular}

\section{b. Evaluaciones de la actuación del BID con fomento a clústers}

En la mayoría de los países latinoamericanos, incluido Brasil, las iniciativas clúster nacionales y regionales contaron con respaldo técnico y financiero del BID. Como además su actuación a este nivel se extendió durante un largo período, es importante considerar algunas de las evaluaciones recientemente realizadas por esta institución sobre las iniciativas apoyadas (Casaburi, Maffioli, Pietrobelli, 2014; Maffioli, 
Pietrobelli, Stucchi, 2016). Las conclusiones siguientes se basan principalmente en esta bibliografía. Es importante aclarar que las intervenciones del BID se concentraron en gran medida en clústers competitivos y ya consolidados.

\section{$\underline{\text { Impactos }}$}

- De acuerdo a estas evaluaciones, los programas de desarrollo de clústers apoyados por el BID tuvieron impactos positivos sobre la eficiencia, el empleo y las exportaciones de las firmas localizadas en clústers, y también generaron externalidades positivas sobre firmas no pertenecientes a los clústers pero vinculadas territorialmente al mismo.

- Por otro lado, estas iniciativas parecen haber contribuido fuertemente a ampliar las interacciones y la coordinación entre los actores locales, así como entre éstos y las agencias públicas regionales y nacionales. Es decir, lograron un incremento de la cooperación privada-privada, público-privada y pública-pública. Hubo también un aumento de las acciones conjuntas y de la cooperación entre empresas, en muchos casos evidenciada en la creación de nuevas asociaciones empresarias.

\section{Limitaciones}

- En primer lugar, las distintas evaluaciones del BID destacan las escasa coordinación lograda entre las iniciativas clústers y el resto de políticas nacionales, incluidas las políticas industriales y de competitividad.

- Dado que el proceso de construcción de relaciones de confianza y cooperación lleva tiempo, el largo plazo de maduración de algunos proyectos conlleva el riesgo de frustración de los actores involucrados.

- Finalmente, se observan dificultades de varios clústers para consolidar y mejorar su inserción en cadenas globales de valor, aunque se reconoce que los clústers pueden justamente hacer una contribución importante para que las empresas logren progresos en ese sentido (Pietrobelli, 2016).

- La selección de los clústers que serán beneficiados por las políticas es una cuestión compleja orientada por las prioridades de desarrollo nacional y regional, pero se debiera considerar tanto la competitividad actual como el potencial de desarrollo de los clústers y la existencia de capacidades mínimas de coordinación.

- Las evaluaciones de impacto rutinarias son imprescindibles para permitir procesos de rectificación y aprendizaje.

\section{$\underline{\text { Aspectos claves de la experiencia BID con fomento a clústers }}$}

\begin{tabular}{|c|c|c|}
\hline Impactos & Limitaciones & Lecciones \\
\hline $\begin{array}{l}\text { Positivos sobre eficiencia, } \\
\text { empleo y exportaciones de } \\
\text { firmas en clústers }\end{array}$ & $\begin{array}{l}\text { Escasa coordinación de } \\
\text { iniciativas clústers y el resto de } \\
\text { políticas nacionales }\end{array}$ & No seguir modelo único \\
\hline $\begin{array}{l}\text { Externalidades positivas sobre } \\
\text { firmas del territorio }\end{array}$ & $\begin{array}{l}\text { Proyectos de larga maduración } \\
\text { pueden provocar frustración y } \\
\text { desistencia de actores }\end{array}$ & $\begin{array}{l}\text { Capacitación previa y } \\
\text { conformación de una gobernanza } \\
\text { clara son factores que aseguran } \\
\text { sustentabilidad }\end{array}$ \\
\hline \multirow[t]{2}{*}{$\begin{array}{l}\text { Incremento de la cooperación } \\
\text { privada-privada, pública-privada } \\
\text { y pública-pública (distintos } \\
\text { niveles) }\end{array}$} & \multirow[t]{2}{*}{$\begin{array}{l}\text { Resultados modestos en cuanto a } \\
\text { inserción de clústers en cadenas } \\
\text { globales de valor }\end{array}$} & $\begin{array}{l}\text { La planificación estratégica } \\
\text { participativa es esencial para } \\
\text { identificar demandas y generar } \\
\text { confianza. }\end{array}$ \\
\hline & & Necesaria coordinación con otras \\
\hline
\end{tabular}




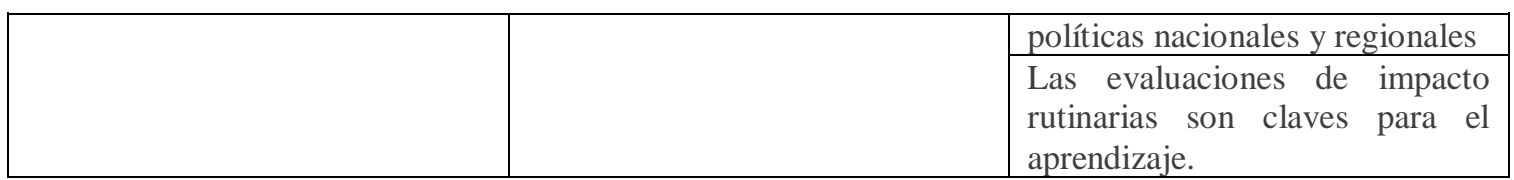

\section{c. Proyecto Polos de Competividad - EU-LAC Foundation}

Finalmente, para completar el panorama sobre las experiencias clústers latinoamericanas, presentaremos algunas conclusiones útiles que surgen del Proyecto "Polos de Competitividad", lanzado en 2014 por la "EU-LAC Foundation", con el objetivo de profundizar la integración de cadenas de valor birregionales (https://eulacfoundation.org/es/competitividad-e-innovación).

Esta iniciativa buscó en un primer momento identificar, en cada país de América Latina y el Caribe y de la Unión Europea, clústers competitivos interesados en aumentar el grado de internacionalización y ampliar la cooperación con clústers complementarios de otra región (ALC o UE). Instituciones públicas y privadas involucradas en el fomento a clústers en cada país prepararon entonces los perfiles de competitividad y de internacionalización de sus respectivos clústers, con el objetivo de implementar diversas acciones buscando aproximar a los polos productivos seleccionados. Participaron en total 10 países latinoamericanos y caribeños, que en total seleccionaron un total de 40 clústers.

Sobre la base de la información provista por los distintos países, se realizó un ejercicio de sistematización de experiencias (EU-LAC Foundation, 2015), que usaremos aquí para extraer las principales conclusiones sobre experiencias clúster en ALC.

\section{$\underline{\text { Impactos }}$}

- El desarrollo de clústers en ALC ha permitido, en términos generales, la difusión de una cultura de cooperación a nivel local y una mejora de la competitividad empresarial. Esto último como resultado de mejoras tecnológicas y mayor eficiencia de los procesos productivos.

- Las políticas de fomento a clústers han favorecido una mayor interacción entre los actores, el desarrollo empresarial y tecnológico y el fortalecimiento de las instituciones empresariales y de apoyo.

\section{Limitaciones}

- El déficit de capital social, es decir la falta de confianza y trayectoria de cooperación entre instituciones públicas y privadas, como también entre empresas, ha limitado el desarrollo de clústers en algunas países/regiones y limitado el impacto de las políticas.

- En muchos clústers se han conseguido establecer prácticas cooperativas para facilitar actividades tales como: compra de materias primas, consorcios de exportación, misiones a ferias y mercados externos, capacitación, certificación de calidad. Pero hay menos avances cooperativos en áreas como innovación (salvo los vínculos entre empresas individuales e instituciones tecnológicas) y comercialización. 
- La excesiva centralización del sistema político-administrativo y las limitadas competencias y recursos de las instancias sub-nacionales pueden restringir su autonomía financiera y su capacidad técnica para definir y gestionar sus propios instrumentos de promoción, siendo que el desarrollo de clústers requiere políticas regionales eficaces y específicas.

- Más allá de los problemas endógenos, los clústers enfrentan grandes déficits de logística, infraestructura y acceso adecuado a financiamiento, todo lo cual demanda mayor coordinación entre las distintas esferas institucionales y políticas.

- La mayoría de los clústers de ALC están en las primeras etapas de consolidación y no tienen estrategias estructuradas de internacionalización (con la importante excepción de México que, debido al NAFTA y a su proximidad con el mercado USA, posee numerosos clústers con estrategias y flujos consolidados de exportación). Además enfrentan obstáculos diversos para internacionalizarse, como déficits en calidad y estandarización, bajas escalas, poca cultura de internacionalización, problemas logísticos, etc. Las políticas no siempre prestan apoyo específico para la superación de cada uno de estos problemas.

Aspectos claves en políticas de clústers de ALC identificados en el Proyecto "Polos de Competitividad" - EU LAC Foundation

\begin{tabular}{|c|c|c|}
\hline Impactos & Limitaciones & Lecciones \\
\hline Mejora competitiva & Déficit de capital social & $\begin{array}{l}\text { Sin gobernanza estructurada no } \\
\text { hay garantía de continuidad de la } \\
\text { iniciativa }\end{array}$ \\
\hline Mayor cooperación & $\begin{array}{lcr}\text { Escasa cooperación } & & \text { en } \\
\text { actividades innovativas y } & \text { de } \\
\text { comercialización interna } & \text { y } \\
\text { externa } & & \end{array}$ & \begin{tabular}{lllr} 
No confundir & \multicolumn{2}{c}{ clústers } & con \\
gestores de & los clústers: \\
prioridad a & proyectos que \\
beneficien & al clúster en & su \\
conjunto & & &
\end{tabular} \\
\hline \multirow[t]{3}{*}{$\begin{array}{l}\text { Fortalecimiento de instituciones } \\
\text { empresariales y de apoyo }\end{array}$} & $\begin{array}{l}\text { Excesiva centralización política- } \\
\text { administrativa de algunos países, } \\
\text { y limitadas capacidades y } \\
\text { recursos de autoridades sub- } \\
\text { nacionales }\end{array}$ & $\begin{array}{lcr}\text { Necesidad } & \text { de } & \text { fortalecer } \\
\text { instancias } & \text { regionales } & \text { para } \\
\text { implementar estas políticas } & \end{array}$ \\
\hline & $\begin{array}{l}\text { Déficits de infraestructura y } \\
\text { acceso a financiamiento limitan } \\
\text { competitividad de clústers }\end{array}$ & $\begin{array}{l}\text { Necesidad de aportar visión } \\
\text { estratégica sobre oportunidades } \\
\text { del clúster en mercado nacional } \\
\text { e internacional, y más en general } \\
\text { una estrategia } \\
\text { internacionalización, inserción } \\
\text { en cadenas de valor, etc. }\end{array}$ \\
\hline & $\begin{array}{l}\text { Maduración incipiente, déficits } \\
\text { tecnológicos y ausencia de } \\
\text { estrategias } \\
\text { internacionalización de } \\
\text { inserción en mercados externos }\end{array}$ & $\begin{array}{l}\text { Atracción de grandes empresas } \\
\text { nacionales y/o extranjeras puede } \\
\text { ser oportunidad para } \\
\text { dinamización tecnológica y } \\
\text { comercial. }\end{array}$ \\
\hline
\end{tabular}

\section{Contribuciones para una nueva agenda de estrategias clúster en América Latina}

En esta sección nos proponemos, en primer lugar, hacer una síntesis de las principales lecciones de las experiencias de políticas clúster ensayadas en la región, que aparecen 
en las evaluaciones y estudios antes presentados. Y en segundo lugar conectar estas enseñanzas con las nuevas tendencias en políticas clúster observadas en la Unión Europea y en algunas experiencias recientes de la región latinoamericana. De este modo podremos acercarnos, a modo de conclusión, a un conjunto de proposiciones claves que pueden servir de referencia para el diseño de una nueva agenda de estrategias clúster en la región. Nuestra premisa es que esta nueva agenda deberá contribuir a, y ser consistente con, el necesario proceso de cambio estructural en América Latina.

\section{a. Principales lecciones de las experiencias recientes}

En los documentos consultados (de muy variada procedencia institucional) surgen varias consideraciones y lecciones coincidentes, y otras que sin ser consensuales son de todos modos muy significativas. Sobre esa base proponemos a continuación una síntesis estilizada de las experiencias de la región con políticas clústers:

- Las políticas de clústers han favorecido efectivamente una mayor cooperación, innovación y competitividad empresarial en estos ecosistemas productivos, y en particular han contribuido a la superación de las restricciones que enfrentan muchas Pymes alli localizadas para crecer e internacionalizarse. También estas políticas, a través del apoyo a clústers más incipientes y en regiones menos industrializadas, han contribuido a superar desigualdades regionales y a lograr una mayor inclusión social.

- Por lo tanto debieran ser un componente importante y permanente de las políticas de cambio estructural, en la medida que los clústers pueden contribuir a disminuir las brechas de productividad y a una mayor incorporación de conocimiento y actividades de base tecnológica. Sin embargo, hasta el presente, las estrategias clúster han sido efectivamente jerarquizadas en pocos países latinoamericanos: en muchos casos su implementación ha sido fragmentaria y transitoria y en otros directamente ni siquiera han sido consideradas dentro del menú de políticas industriales.

- Como resultado de lo anterior, y en línea con la configuración de la estructura productiva latinoamericana, gran parte de los clústers operan en actividades vinculadas al procesamiento de recursos naturales, presentan baja capacidades empresariales y de innovación, déficits de calidad y productividad, problemas de gobernanza... Es decir, hay una amplia agenda vacante para las estrategias clúster.

- Las políticas de clúster implementadas en la región han tenido impactos positivos: han puesto en valor las intervenciones a nivel territorial y en términos generales contribuyeron a consolidar gobernanzas e intensificar las interacciones entre los actores del clúster, públicos y privados, así como a la coordinación de políticas a nivel local y regional. También favorecieron la absorción de conocimiento y el upgrade tecnológico, la capacitación empresarial, el acceso a mercados y en algunos casos un aumento de las exportaciones.... Como resultado, las políticas contribuyeron al crecimiento económico de las empresas y del empleo.

- Sin embargo, las políticas clúster generalmente se desarrollaron en forma aislada, es decir, sin coordinación ni consistencia con otras políticas nacionales y regionales, que son también relevantes para el éxito de los clúster, como las políticas industriales y sectoriales, de infraestructura, de formación, C\&T, etc. 
- En particular, muchas de las iniciativas clúster no se han podido conectar adecuadamente con el contexto y prioridades más amplias del desarrollo local y regional, en alguna medida porque en muchos casos simplemente no existían estrategias de innovación y desarrollo regional.

- Es importante entonces que las estrategias clúster estén fuertemente asociadas al resto de políticas de nivel nacional y regional, y particularmente a las políticas industriales, de innovación y de infraestructura.

- Las estrategias clúster son relativamente nuevas, específicas y complejas, y por lo tanto requieren ser dotadas de institucionalidades -nacionales, regionales y locales- e instrumentos acordes con la naturaleza sistémica y colectiva de la intervención. No es pertinente ni eficaz la aplicación exclusiva de instrumentos horizontales "de uso general" en política industrial. Esto vale para los mecanismos de acceso al crédito, para los incentivos a la innovación y la internacionalización, etc.

- Debido a la gran heterogeneidad de estructuras productivas regionales y clústers, y a la diversidad de estadios de maduración institucional/tecnológica/productiva, las políticas clúster deben ser flexibles y no seguir un modelo único.

- Se pueden observar algunas "fallas" recurrentes de las políticas, señaladas en las experiencias previas, como ser:

○ "encapsulamiento" de la estrategia clúster que aparece desvinculada del contexto más general de políticas de desarrollo nacional y regional,

○ priorización del apoyo "al gestor del clúster" en desmedro del apoyo "al clúster",

- dificultad para asegurar la sostenibilidad de las actividades de fomento y gestión del clúster una vez finalizado el apoyo público,

○ incumplimiento de las rutinas de evaluación periódica...

- Para finalizar, pueden señalarse algunos componentes importantes incorporados en algunos casos pero que no siempre fueron incluidos en el menú clásico de políticas de años recientes en la región:

- apoyo el desarrollo de estrategias efectivas de internacionalización de los clústers más maduros y competitivos,

○ promoción de encadenamientos productivos y transferencias tecnológicas entre clústers conexos (tanto a nivel regional, nacional e internacional),

- apoyo al desarrollo de una inteligencia estratégica de los clústers en los segmentos de mercado en que actúan,

- promoción activa de alianzas y eventualmente atracción de empresas extranjeras o de grandes firmas que impacten favorablemente en el proceso de maduración e internacionalización de los clústers, etc.

\section{b. Nuevas tendencias en políticas de clúster}

La política de clúster se ha convertido desde hace ya varios años en un elemento importante de las políticas de innovación y competitividad en los países y regiones de Europa. Y en el marco de la UE hay diversas iniciativas que buscan dar impulso y potenciar a estos polos productivos y de innovación.

En la Estrategia "Europa 2020", que es el actual marco de referencia para la estrategia de crecimiento de la Unión Europea, los clústers mantienen ese papel fundamental. Pero al mismo tiempo las estrategias regionales de innovación pasaron a jugar un rol muy 
importante y creciente, siendo que las políticas de clústers continúan a ser un componente clave pero no único de las mismas.

Efectivamente, y en línea con el "crecimiento inteligente" perseguido por la nueva estrategia europea, desde hace algunos años se han movilizado grandes incentivos para que las regiones europeas desarrollen "Estrategias de Especialización Inteligente" (conocidas como RIS3 - Research and Innovation Strategies for Smart Specialization-), cuya idea central reside en concentrar recursos en las áreas con ventajas comparativas y competitivas más prometedoras de cada región.

De acuerdo con Castillo, Barroeta, Paton (2011), el concepto de especialización inteligente tiene su origen en la reflexión generada en la UE a principios de los años 2000, en torno a la brecha de productividad y de capacidad de innovación con USA, que aparecía como resultado de una menor especialización tecno-productiva y también de una menor capacidad de priorización de esfuerzos a nivel regional en Europa. Al mismo tiempo, la crisis europea reciente impuso una lógica de economía y priorización de recursos y favoreció un enfoque que buscaba evitar la sobreposición de esfuerzos y de iniciativas tecno-productivas en las regiones.

Los autores mencionados observan que, respecto a la anterior conceptualización de estrategias de innovación regional en la UE, las actuales RIS3 enfatizan la importancia de la priorización de una especialización basada en KETs (Key Enabling Technologies, es decir nuevas tecnologías de impacto transversal) que tenga en cuenta la priorización de otras regiones (para evitar sobre-posiciones), la formulación de un proceso de gobernanza participativa, y la evaluación y seguimiento para garantizar la transparencia y la mejora continua.

Es decir que las RIS3 plantean, más allá de la necesaria priorización de esfuerzos en áreas con masa crítica y claras ventajas competitivas, que esa inteligencia estratégica debe surgir de un "proceso de descubrimiento" a nivel territorial que involucre una amplia gama de actores. En este sentido las estrategias regionales de innovación dejan de ser visualizadas como meras estrategias de gobierno, para ser encaradas como verdaderas estrategias de los territorios y que involucran un proceso de descubrimiento en el que participan todos los actores de la llamada "cuádruple hélice" (gobierno, empresas, universidad y sociedad civil) (Barroeta, Paton, Castillo, 2012).

Vemos entonces que hay una serie de conceptos y lógicas en las RIS3 que en buena medida ya eran inherentes a las políticas de clústers: enfoque sistémico, eje en la innovación y el aprendizaje interactivo, cooperación, valoración del territorio como espacio privilegiado para la generación y absorción de conocimiento tácito y donde se construyen ventajas competitivas, énfasis en emprendedorismo, etc. Además, los clústers son componentes claves de las RIS3, porque constituyen las bases de especialización y competitividad empresarial de las regiones, porque en muchos casos son justamente los proveedores de tecnologías horizontales que las RIS3 se proponen diseminar, y porque expresan coaliciones de actores que, aunque específicos, son también los que se proponen movilizar las estrategias regionales (European Commission, 2013).

Es decir que las RIS3 no significan una discontinuidad respecto a las políticas de clústers sino más bien una ampliación de su horizonte, en la medida que incorporan 
otros niveles y escalas de políticas (sectoriales, educacionales, culturales...) y actores regionales que no necesariamente están directamente vinculados con los clústers. El alcance de las RIS3 es más amplio que el de las políticas clústers, pero hay claras sinergias entre ambas estrategias: los clústers tienen un rol fundamental evidente para el éxito de las RIS3, al tiempo que éstas ofrecen a los clústers la posibilidad de contar con un ámbito mayor y más eficiente para buscar soluciones a temas horizontales y sectoriales (como ser la formación de recursos humanos, inversiones en C\&T\&I, infraestructura y logística), e incluso para articularse entre sí (clústers de la misma región).

El enfoque RIS3 inspiró algunas iniciativas recientes en América Latina, que tuvieron como objetivo el impulso a agendas regionales de innovación pero que también incorporaron componentes de fomento a clústers.

En Brasil, en 2017 fue lanzado en el Estado de Pernambuco el Proyecto RIS3-PE (Estrategias de Especialización Inteligente en Territorios Innovadores Seleccionados), una iniciativa piloto que puede llevar a la implementación de otras RIS3 en ese país. El Proyecto RIS3-PE está alineado con la "Estrategia de Ciencia, Tecnología e Innovación para Pernambuco 2017-2022”, que contempla también el fortalecimiento e integración de hábitats de innovación, como los APLs de ese Estado.

En 2014, el gobierno de México lanzó la iniciativa "Agendas Estatales y Regionales de Innovación, con el objetivo de articular y definir prioridades sectoriales y áreas de especialización inteligente e impulsar proyectos estratégicos de innovación a nivel de las regiones. Inspiradas explícitamente en las RIS3 implementadas en la UE, estas Agendas “...son procesos de intervención que ayudan a los estados y regiones a enfocar estratégicamente los recursos disponibles para detonar y apoyar proyectos de alto impacto. Éstas se enfocan principalmente en las capacidades específicas y la vocación económica de cada entidad y región, con miras a desarrollar su potencial para innovar y competir en el contexto regional, nacional y global"(http://www.agendasinnovacion.org/). Cada Estado mexicano cuenta actualmente con una Agenda propia, y parte importante de las mismas consiste en apoyar a los distintos clústers en sus proyectos de innovación

Por su lado, Chile lanzó en 2015 los "Programas Estratégicos de Especialización Inteligente" para favorecer una mayor diversificación y sofisticación de su matriz productiva. La iniciativa abarca diversos sectores productivos y actividades tecnológicas y de servicios (Minería, Pesca y acuicultura, Industria inteligente, Manufactura avanzada, Logística, etc), y consiste básicamente en la organización de instancias de coordinación público-privadas para seleccionar e impulsar proyectos de innovación, infraestructura y reestructuración productiva a nivel nacional pero también también regional. Es decir, la iniciativa busca mejorar los desempeños innovativos y productivos en sectores y territorios específicos. Como en el caso mexicano, aquí también el fortalecimiento de diversos clústers -claves en actividades como salmón, minería y TIC-, forma parte de la iniciativa.

Finalmente, la estrategia de apoyo a Clústers en Colombia, liderada por INNpulsa (agencia vinculada al Ministerio de Comercio, Industria y Turismo, encargada de promover el desarrollo empresarial a través del emprendimiento, la innovación y la productividad), también viene incorporando la perspectiva regional y de especialización 
inteligente en todas sus iniciativas clústers. Como parte de esa nueva orientación, se destacan las iniciativas de vinculación interclúster dentro de las regiones y el nuevo rol asignado a los Clústers de TI de fertilización tecnológica horizontal (iNNpulsa Colombia, 2018).

\section{Conclusiones}

Para retomar y hacer sustentables los procesos de crecimiento con inclusión social que varios países de América Latina protagonizaron desde principios de este siglo y hasta recientemente, y también para hacer frente a los desafíos planteados por la revolución tecnológica e industrial en curso, será preciso que la región impulse profundos procesos de transformación, diversificación y sofisticación productiva. Ello supone, por un lado, superar las enormes brechas de productividad y la heterogeneidad estructural de nuestras economías, a través de la modernización de sectores productivos tradicionales y amplios segmentos empresariales Pymes que operan típicamente con baja eficiencia. Por otro, supone un proceso de incorporación de conocimientos y de nuevas actividades de alto valor agregado. Se trata de pasar de una especialización productiva y exportadora basada en ventajas estáticas, a otra basada en ventajas dinámicas e innovativas.

Las políticas industriales y tecnológicas son fundamentales para viabilizar ese proceso, y entre ellas se destacan las políticas de clústers. Como instrumentos probadamente aptos para impulsar la cooperación, la innovación y la competitividad empresarial particularmente en Pymes-, pueden hacer una contribución importante al proceso de cambio estructural. También, en la medida que permitan el desarrollo de clústers innovadores y competitivos, podrán favorecer una mayor y mejor inserción de estos sistemas productivos especializados en el mercado internacional y en cadenas globales de valor.

Para ello será necesario considerar las principales enseñanzas que se desprenden de la experiencia reciente.

En primer lugar, las políticas de clúster debieran pasar a tener continuidad y una mayor jerarquía en las agendas públicas de la región que las observadas hasta el presente en la mayor parte de países de la región.

En segundo lugar, debido a la heterogeneidad y especificidad de cada región/sector/clúster, no parece apropiado seguir modelos únicos de fomento a los mismos. A su vez, es útil diferenciar las intervenciones en función de la taxonomía de agrupamientos productivos presentes. Existen clústers de distinta naturaleza (dada por su estructura, grado especialización, dinamismo tecnológico, etc), y que a a su vez transitan distintos estadios madurativos. Las políticas para clústers más incipientes y en actividades tradicionales de bajo dinamismo innovativo, no pueden asimilarse a las que requieren clústers competitivos, en sectores de alta tecnología y con posibilidades de una inserción dinámica en el mercado internacional.

En tercer lugar, será preciso que se articulen fuertemente con el conjunto de políticas nacionales orientadas a la transformación productiva y la competitividad, puesto que el 
desempeño de los clústers depende también de factores exógenos (sectoriales, de infraestructura y logística, etc).

Finalmente, es importante que las políticas de clúster formen parte activa de estrategias más amplias de innovación regional, para aprovechar las sinergias y las potencialidades que puede aportar esa convergencia de políticas. En ese sentido también es clave fortalecer las capacidades regionales para la implementación de estas iniciativas.

\section{Bibliografía}

Barroeta B., Paton J., Castillo J. (2012): Instrumentos de política RIS3 Clústers, INFYDE, Working Paper Series, Nº6, Biscaia.

Becattini G.: From Marshall's to the Italian "Industrial Districts". A Brief Critical Reconstruction. In: Complexity and Industrial Clusters. Dynamics and Models in Theory and Practice, Curzio A. y Fortis M. Ed., 2002. Physica-Verlag Heidelberg, Berlin, 2002.

Boscherini F., Poma L. (ed.) (2000): Territorio, conocimiento y competitividad de las empreas: el rol de las instituciones en el contexto global, Editorial Miño y Dávila, Buenos Aires.

Casaburi, G., Maffioli, A., Pietrobelli, C. 2014. More than the Sum of its Parts: Cluster-Based Policies, en G. Crespi, E. Fernandez-Arias, y E. H. Stein, eds., Rethinking Productive Development: Sound Policies and Institutions for Economic Transformation. Washington, DC: Palgrave Macmillan y Banco Interamericano de Desarrollo.

Cassiolato, J. E., Matos, M. P. : Política brasileira para arranjos produtivos locais: o aprendizado acumulado e suas perspectivas. In: Lastres, H. M. M. et al. (Org.). A nova geração de políticas de desenvolvimento produtivo: sustentabilidade social e ambiental. Brasília: Editora da CNI, 2012.

Castillo J., Barroeta B., Paton J. (2011): Estrategias Regionales de Especialización Inteligente (RIS 3). GUÍA RÁPIDA, INFYDE Working Paper Series, Año 2 Vol. 1 No.5, Biscaia.

CEPAL (2016): Horizontes 2030: la igualdad en el centro del desarrollo sostenible, Santiago de Chile.

CEPAL (2017): Brechas, ejes y desafíos en el vínculo entre lo social y lo productivo, Santiago de Chile.

CEPAL (2018): La ineficiencia de la desigualdad, Santiago de Chile.

Cravo T., Lodato S., Piza C. (2013): Industrial clusters and economic performance in Brazil, IDB Working Paper Series ; 475, IDB. 
EU-LAC Foundation (2015): Shared experiences on competitiveness and internationalisation of competitive territories in the EU and LAC countries, Project Report, EU LAC Foundation (web: http://polos.eulacfoundation.org/system/files/Competitive\%20and\%20internationa $1 \%$ 20territories.pdf).

European Commission (2013): The role of clusters in smart specialisation strategies, Directorate-General for Research and Innovation.

iNNpulsa Colombia, Clúster Development (2018): Iniciativas Clúster en Colombia, Bogotá.

Lastres H., C. Pietrobelli C., Caporali R., Soares M, Matos M. (org.) (2012): A nova geração de políticas de desenvolvimento produtivo: sustentabilidade social e ambiental, CNI, Brasilia.

Maffioli A., Pietrobelli C., Stucchi R. (ed) (2016):. The Impact Evaluation of Cluster Development Programs. BID, Washington D.C.

Matos M.P. , Borin E. , Cassiolato J.E. (org) (2015): Uma década de evolução dos arranjos produtivos locais , E-Papers, Rio de Janeiro.

Matos M.P., Borin E., Cassiolato J.E., Arruda D., Marcellino I.S. (2016): Brasil: uma década de políticas para arranjos produtivos locais e sua evolução, Revista Pymes, Innovación y Desarrollo, Ene-Abr, Vol. 4, No.1, pp. 11-36.

Matos M.P., Cassiolato J.E., Lastres H.M.M., Lemos C., Szapiro M. (orgs) (2017): APLs: referencial, experiências e políticas em 20 anos da RedeSist. E-Papers, Rio de Janeiro.

Pietrobelli C. (2015): ¿Qué lecciones ha aprendido América Latina y el Caribe en materia de políticas de clústers? https://blogs.iadb.org/Ideasquecuentan/2015/09/21/que-lecciones-ha-aprendidoamerica-latina-y-el-caribe-en-materia-de-politicas-de-clústers/

Pietrobelli C.: (2016): Políticas industriales modernas para el cambio estructural en América Latina: coordinación e integración productiva. Boletín Techint $\mathrm{N}^{\mathrm{o}} 353$, Buenos Aires.

Pietrobelli, C., Rabellotti R. (2007). Upgrading to Compete: Clústers and Value Chains in Latin America. Cambridge, MA: Harvard University Press.

Porter, M.E. 1998. Clusters and the New Economics of Competition. Harvard Business Review, Noviembre-Diciembre.

Schmitz H. (1997): Eficiência coletiva: caminho de crescimento para a indústria de pequeno porte. Ensaios FEE, V. 18, N. 2, Porto Alegre.

Schmitz H., Nadvi K. (1999): Clustering and Industrialization: Introduction. World Development, vol. 27. 